\title{
Mathematical identification of a neuronal network consisting of GABA and DA in striatal slices of the rat brain
}

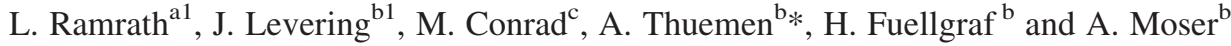 \\ ${ }^{a}$ Institute for Robotics und Cognitive Systems, University of Luebeck, D-23538 Luebeck, Germany; \\ ${ }^{b}$ Neurochemical Research Group, Department of Neurology, University of Luebeck, D-23538 Luebeck, \\ Germany; ' Institute of Mathematics, University of Luebeck, D-23538 Luebeck, Germany
}

(Received 9 May 2008; final version received 11 November 2008)

\begin{abstract}
High frequency stimulation (HFS) has been used to treat various neurological and psychiatric diseases. Although further disorders are under investigation to extend the clinical application of HFS, the complex effect of HFS within a neuronal network is still unknown. Thus, it would be desirable to find a theoretical model that allows an estimation of the expected effect of applied HFS. Based on the neurochemical analysis of effects of the $\gamma$-aminobutyric acid $(\mathrm{GABA})_{A}$ receptor antagonist bicuculline, the D2-like receptor antagonist sulpiride and the D1-like receptor antagonist SCH-23390 on HFS evoked GABA and dopamine (DA) release from striatal slices of the rat brain, a mathematical network model is proposed including the neurotransmitters GABA, DA and glutamate (GLU). The model reflects inhibitory and excitatory interactions of the neurotransmitters outflow in the presence of HFS. Under the assumption of linear interactions and static measurements, the model is expressed analytically. Numerical identification of inhibition and excitation is performed on a basis of real outflow levels of GABA and DA in the rat striatum. Results validate the nature of the proposed model. Therefore, this leads to an analytical model of the interactions within distinct neural network components of the rat striatum.
\end{abstract}

Keywords: high frequency stimulation; neural network; analytical model; striatum

2000 Mathematics Subject Classifications: 93B30; 92B20; 93A30; 92C20; 93C05; 15A06

\section{Introduction}

In clinical practice, it is well known that the high frequency stimulation (HFS) in different locations of cortico-striato-thalamico-cortical loops alleviates symptoms of neurological and psychiatric disorders, e.g. Parkinson's disease, dystonias, obsessive compulsive disorders, Tourette's syndrome, Huntington disease, epilepsy [3,4,6,10,21]. Target areas for electrical HFS are the thalamus, the internal globus pallidus (GPi), the subthalamic nucleus or the caudate nucleus $[3,5,6,10,14]$. Despite the positive effects of HFS, we do not know much about the biochemical mechanisms and interactions of HFS within the respective integrated neuronal networks $[8,11,22]$. Thus, there exists no rationale to assess precisely the effect of HFS in a pathological imbalanced neuronal network.

Li et al. [16], therefore, established an in vitro model to analyse neurotransmission under HFS in striatal slices of the rat brain. Neurochemical experiments were conducted to exploit the effect of HFS on a neuronal network consisting of GABA, glutamate (GLU) and dopamine (DA)

*Corresponding author. Email: ansgar.thuemen@neuro.uni-luebeck.de 
neurons. HFS with $130 \mathrm{~Hz}$ stimulated veratridine (VER)-activated GABAergic neurons while DA outflow decreased. Outflow of both neurotransmitters increased in the presence of $\mathrm{GABA}_{A}$ receptor antagonist bicuculline. Addition of the competitive DA D2-like receptor antagonist S-(-)-sulpiride caused a decline of HFS-evoked stimulatory effect on GABA outflow to values found after VER without HFS. Coincubation of sulpiride and the competitive D1-like receptor antagonist R-(+)-SCH-23390 completely reversed the effect of sulpiride on HFS plus VERinduced GABA outflow. In all experiments, no HFS-effect could be observed on GLU outflow. Quantitative results of the measurement results in Ref. [16] are given in Table 1 and the neurochemical model of HFS effects on a neuronal network is summarized in Figure 1. The results emphasized that HFS primarily affects GABAergic neurons in vitro. Since these neurons are embedded in a neuronal network with a GABA-DA circuit, HFS would interact with a neuronal network and not only with one neurotransmitter system or one neuron population. Based on these in vitro results, $\mathrm{Li}$ et al. [16] suggested that HFS primarily affects GABAergic neurons which are embedded in a neural network with a GABA-DA circuit, without any changes in GLU activity.

This work concentrates on the theoretical modelling of HFS-evoked interactions within a neural network consisting of two or three, excitatory (e.g. DA and GLU) and inhibitory (e.g. GABA) neurons. Mutual influences of respective neurons are modelled in order to determine the total network action. Interactions of the neurons under HFS are assumed to be linear. The analysis is based on quantitative measurements obtained in Ref. [16] and numerical identification of interaction coefficients is performed.

This contribution is organized as follows: In Section 2, a model consisting of only dopaminergic and GABAergic neurons is introduced and parameterized. As results do not show sufficient correlation of simulated and true measurements, the model is extended to a threeneuron model in Section 3. Two variations of a three-neuron model are subsequently parameterized and compared to the true measurements. Models are analysed under the assumption of direct and indirect effects of HFS. In Sections 4 and 5, results are discussed and a conclusion is drawn.

\section{Two-neuron model}

\subsection{Modelling}

Analysing the GABA and DA outflow under different influence of HFS and the different antagonists, a two-neuron interaction model can be proposed since GLU levels remained unchanged under different experimental conditions. The following relations are derived from an interpretation of the measurement results:

Table 1. GABA, DA, GLU outflow from striatal slices under different conditions as indicated. Values are expressed in $\mathrm{nM} \pm \mathrm{SD}$. GABA: $\gamma$-aminobutyric acid, DA: dopamine, GLU: glutamate, VER: veratridine, HFS: high frequency stimulation, BIC: bicuculline, SLP: sulpiride, SCH: SCH-23390.

\begin{tabular}{lrcl}
\hline Scenario & GABA $(\mathrm{nM})$ & DA $(\mathrm{nM})$ & GLU $(\mathrm{nM})$ \\
\hline BASAL & $6.40 \pm 2.76$ & $17.69 \pm 2.90$ & $34.26 \pm 8.27$ \\
VER & $14.10 \pm 5.45$ & $25.52 \pm 4.42$ & Not changed \\
VER + HFS & $19.54 \pm 3.80$ & $18.00 \pm 3.00$ & Not changed \\
VER + HFS + BIC & $30.70 \pm 7.70$ & $25.50 \pm 5.70$ & Not changed \\
VER + HFS + SLP & $13.76 \pm 2.37$ & $18.00 \pm 3.00$ & Not changed \\
VER + HFS + SLP + SCH & $18.69 \pm 3.39$ & $18.50 \pm 4.00$ & Not changed \\
\hline
\end{tabular}




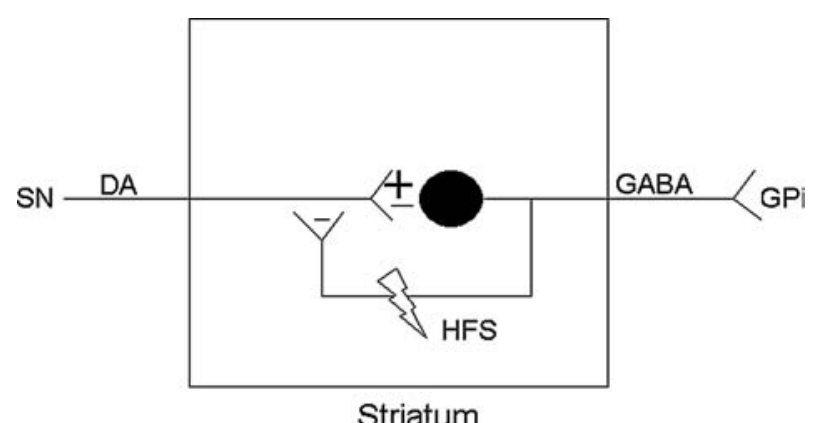

Figure 1. HFS effect on a GABA-DA circuit (from Li et al. [16]). GPi: internal globus pallidus, SN: substantia nigra; GABA: $\gamma$-aminobutyric acid; DA: dopamine.

(i) Increasing GABA outflow induces self-inhibition via $\mathrm{GABA}_{A}$ receptors resulting in decreasing GABA outflow.

(ii) Increasing GABA outflow diminishes the DA outflow via the presynaptic $\mathrm{GABA}_{A}$ receptors.

(iii) Increasing DA outflow has heterogeneous effects: excitatory and inhibitory effects on the GABAergic system via D1- and D2-like receptors, respectively.

(iv) Increasing DA outflow induces self-inhibition via D1-like receptors resulting in decreasing DA outflow.

(v) VER is assumed to enhance GABA and DA outflow.

(vi) HFS primarily affects GABAergic neurons and is assumed to enhance GABA outflow.

These assumptions motivate a neurotransmitter dependence model as shown in Figure 2. Excitation and inhibition are represented by the factors $P$ or $N$, respectively. Constant influences are introduced by factors $k_{1}-k_{4}$.

To build up the mathematical model of GABA and DA interactions under HFS, the neural network shown in Figure 2 is expressed analytically. VER and HFS are assumed as network input. Each connection between two network components is labelled with a factor indicating the effect on neurotransmitter concentration. The neurotransmitter outflow results from the addition

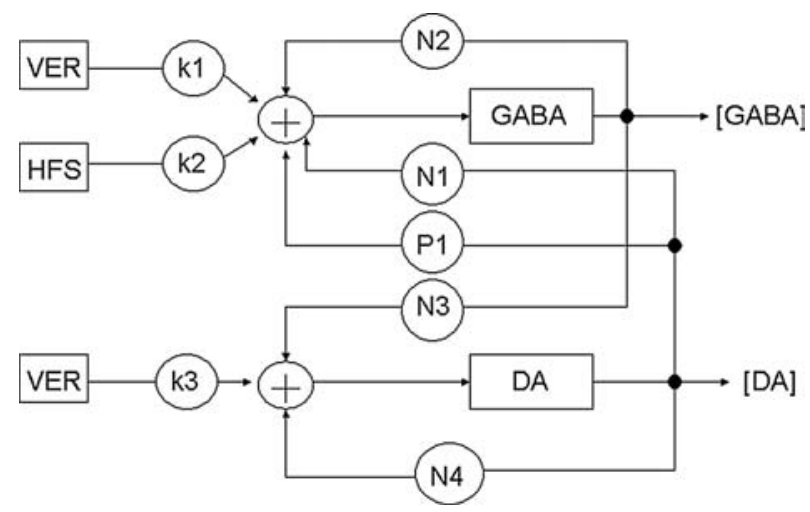

Figure 2. Two-neuron model of GABA-DA relation under HFS. The coefficients of modulation are circled and network components are indicated by rectangles. Inhibition is given by factors $N$, excitation by $P$. 
of all weighted influences. For a mathematical analysis the coefficients are assumed to be timeinvariant.

\subsection{Mathematical identification}

Based on the above described interactions between GABAergic and dopaminergic neurons following HFS, concentrations of GABA and DA conglomerate as shown in the following Equations (1) and (2):

$$
\begin{gathered}
k_{1} \cdot \operatorname{VER}+k_{2} \cdot \mathrm{HFS}+N_{2} \cdot \mathrm{GABA}^{(i)}+N_{1} \cdot \mathrm{DA}^{(i)}+P_{1} \cdot \mathrm{DA}^{(i)}=\mathrm{GABA}^{(i)}, \\
k_{3} \cdot \mathrm{VER}+N_{3} \cdot \mathrm{GABA}^{(i)}+N_{4} \cdot \mathrm{DA}^{(i)}=\mathrm{DA}^{(i)}
\end{gathered}
$$

where $i=1, \ldots, 6$ denotes the respective measurement scenario (Table 1). GABA and DA are subsequently substituted by mean neurotransmitter concentration listed in Table 1 . In case of a given effect, VER $=$ HFS $=1$, otherwise VER and HFS are assumed to be zero. In each different experimental condition, distinct receptor types were blocked by antagonists. Thus, in each measurements different interactions between GABA and DA were suppressed leading to a coefficient set to zero and resulting in a set of dissimilar equations for the GABAergic (Equation (3)) and the dopaminergic system (Equation (4)). The different measurement scenarios therefore establish a set of six equations for the GABAergic system.

$$
\begin{aligned}
N_{2} \mathrm{GABA}^{(1)}+N_{1} \mathrm{DA}^{(1)}+P_{1} \mathrm{DA}^{(1)} & =\mathrm{GABA}^{(1)}, \\
k_{1}+N_{2} \mathrm{GABA}^{(2)}+N_{1} \mathrm{DA}^{(2)}+P_{1} \mathrm{DA}^{(2)} & =\mathrm{GABA}^{(2)}, \\
k_{1}+k_{2}+N_{2} \mathrm{GABA}^{(3)}+N_{1} \mathrm{DA}^{(3)}+P_{1} \mathrm{DA}^{(3)} & =\mathrm{GABA}^{(3)} \\
k_{1}+k_{2}+N_{1} \mathrm{DA}^{(4)}+P_{1} \mathrm{DA}^{(4)} & =\mathrm{GABA}^{(4)} \\
k_{1}+k_{2}+N_{2} \mathrm{GABA}^{(5)}+N_{1} \mathrm{DA}^{(5)} & =\mathrm{GABA}^{(5)} \\
k_{1}+k_{2}+N_{2} \mathrm{GABA}^{(6)} & =\mathrm{GABA}^{(6)},
\end{aligned}
$$

and

$$
\begin{aligned}
N_{3} \mathrm{GABA}^{(1)}+N_{4} \mathrm{DA}^{(1)} & =\mathrm{DA}^{(1)}, \\
k_{3}+N_{3} \mathrm{GABA}^{(2)}+N_{4} \mathrm{DA}^{(2)} & =\mathrm{DA}^{(2)}, \\
k_{3}+N_{3} \mathrm{GABA}^{(3)}+N_{4} \mathrm{DA}^{(3)} & =\mathrm{DA}^{(3)}, \\
k_{3}+N_{4} \mathrm{DA}^{(4)} & =\mathrm{DA}^{(4)}, \\
k_{3}+N_{3} \mathrm{GABA}^{(5)}+N_{4} \mathrm{DA}^{(5)} & =\mathrm{DA}^{(5)}, \\
k_{3}+N_{3} \mathrm{GABA}^{(6)} & =\mathrm{DA}^{(6)},
\end{aligned}
$$

for the dopaminergic neurons. By means of these equations, the five coefficients for the GABAergic and the three for the DAergic system can be determined. An important property of the GABAergic and dopaminergic set of equations is inherent generality. The parameters of interaction in the twoneuron model which are assumed to reflect inhibitory and excitatory effects enter the identification as constants with no restriction on magnitude or sign. Thus, interactions different from the assumptions can result from the identification process. 
For each system, the set of equations were transformed into matrix form

$$
\mathbf{A x}=\mathbf{y}
$$

The matrix A contains the values for VER, HFS, GABA and DA, vector $\mathbf{y}$ the mean concentrations of the neurotransmitter and the vector $\mathbf{x}$ includes the unknown coefficients (Equations (6) and (7)),

$\left[\begin{array}{ccccc}0 & 0 & \mathrm{GABA}^{(1)} & \mathrm{DA}^{(1)} & \mathrm{DA}^{(1)} \\ \mathrm{VER} & 0 & \mathrm{GABA}^{(2)} & \mathrm{DA}^{(2)} & \mathrm{DA}^{(2)} \\ \mathrm{VER} & \mathrm{HFS} & \mathrm{GABA}^{(3)} & \mathrm{DA}^{(3)} & \mathrm{DA}^{(3)} \\ \mathrm{VER} & \mathrm{HFS} & 0 & \mathrm{DA}^{(4)} & \mathrm{DA}^{(4)} \\ \mathrm{VER} & \mathrm{HFS} & \mathrm{GABA}^{(5)} & \mathrm{DA}^{(5)} & 0 \\ \mathrm{VER} & \mathrm{HFS} & \mathrm{GABA}^{(6)} & 0 & 0\end{array}\right]\left[\begin{array}{c}k_{1} \\ k_{2} \\ N_{2} \\ N_{1} \\ P_{1}\end{array}\right]=\left[\begin{array}{c}\mathrm{GABA}^{(1)} \\ \mathrm{GABA}^{(2)} \\ \mathrm{GABA}^{(3)} \\ \mathrm{GABA}^{(4)} \\ \mathrm{GABA}^{(5)} \\ \mathrm{GABA}^{(6)}\end{array}\right]$,

$$
\left[\begin{array}{ccc}
0 & \mathrm{GABA}^{(1)} & \mathrm{DA}^{(1)} \\
\mathrm{VER} & \mathrm{GABA}^{(2)} & \mathrm{DA}^{(2)} \\
\mathrm{VER} & \mathrm{GABA}^{(3)} & \mathrm{DA}^{(3)} \\
\mathrm{VER} & 0 & \mathrm{DA}^{(4)} \\
\mathrm{VER} & \mathrm{GABA}^{(5)} & \mathrm{DA}^{(5)} \\
\mathrm{VER} & \mathrm{GABA}^{(6)} & 0
\end{array}\right]\left[\begin{array}{c}
k_{3} \\
N_{3} \\
N_{4}
\end{array}\right]=\left[\begin{array}{c}
\mathrm{DA}^{(1)} \\
\mathrm{DA}^{(2)} \\
\mathrm{DA}^{(3)} \\
\mathrm{DA}^{(4)} \\
\mathrm{DA}^{(5)} \\
\mathrm{DA}^{(6)}
\end{array}\right]
$$

where $\mathrm{GABA}^{(1)}$ denotes the mean GABA concentration from the first measurement scenario. The linear Equations (6) and (7) are over-determined and, in general, a solution cannot be found. This leads to the standard associated least-squares problem

$$
\|A x-y\|_{2} \rightarrow \min ,
$$

which can be solved by the normal equation

$$
\left(A^{\top} \cdot A\right) x=A^{\top} \cdot y
$$

with the unique solution

$$
x=\left(A^{\top} \cdot A\right)^{-1} \cdot A^{\top} \cdot y,
$$

if the inverse of $\left(A^{\top} \cdot A\right)$ exists. Table 2 shows results for the coefficients if mean concentrations of GABA and DA from Table 1 are used. To demonstrate the quality of the found solution, the relative and the absolute concentration errors are determined. The absolute error is calculated as the absolute value of the difference between the mean concentrations of the measurement results $(y)$ and the calculated model output concentrations $(\hat{y}=A x)$, i.e.

$$
e_{a, \mathrm{GABA}}^{(i)}=\left|y_{\mathrm{GABA}}^{(i)}-\hat{y}_{\mathrm{GABA}}^{(i)}\right|, \quad e_{a, \mathrm{DA}}^{(i)}=\left|y_{\mathrm{DA}}^{(i)}-\hat{y}_{\mathrm{DA}}^{(i)}\right|,
$$

where $y_{\mathrm{GABA}}^{(i)}$ denotes the GABA concentration in the $i$ th measurement scenario. The error values for all six measurement scenarios for both neurotransmitter systems are given in Table 3 . The maximum of all absolute errors is 2.83 for GABA and 6.42 for DA. The relative error results 
Table 2. Coefficients for the GABA and DA system under HFS for the different models. A positive factor shows an excitatory effect, a negative one an inhibitory effect. The second column of GABA and DA coefficients denotes the coefficients for the two-neuron model, the third column the coefficients for the three-neuron model where HFS affects primarily GABAergic neurons, and the fourth column the coefficients for the three-neuron model where HFS affects GABAergic and dopaminergic neurons.

\begin{tabular}{lrrr}
\hline & Two-neuron & Three-neuron 1 & Three-neuron 2 \\
\hline GABA & & & 9.12 \\
$k_{1}$ & 9.12 & 9.12 & 12.29 \\
$k_{2}$ & 12.29 & 12.29 & -0.27 \\
$N_{2}$ & -0.27 & -0.27 & -0.22 \\
$N_{1}$ & -0.22 & -0.22 & 0.56 \\
$P_{1}$ & 0.56 & 0.56 & 11.48 \\
DA & & & -6.48 \\
$k_{3}$ & 7.54 & 5.15 & -0.43 \\
$k_{4}$ & & & -0.05 \\
$N_{3}$ & 0.24 & -0.25 & 0.62 \\
$N_{4}$ & 0.59 & 0.15 & 0.48 \\
$P_{2}$ & & & \\
\hline
\end{tabular}

Table 3. Absolute and relative errors between measurement and model output for the two-neuron model.

\begin{tabular}{lccccc}
\hline & \multicolumn{2}{c}{ GABA } & & \multicolumn{2}{c}{ DA } \\
\cline { 2 - 3 } \cline { 5 - 6 } & Absolute $(\mathrm{nM})$ & Relative & & Absolute (nM) & Relative \\
\hline CTRL & 2.05 & 0.32 & 5.61 & 0.32 \\
VER & 0.00 & 0.00 & 0.63 & 0.02 \\
VER + HFS & 2.83 & 0.14 & 5.00 & 0.28 \\
VER + HFS + BIC & 0.57 & 0.02 & 2.79 & 0.11 \\
VER + HFS + SLP & 0.00 & 0.00 & 3.59 & 0.20 \\
VER + HFS + SLP + SCH & 2.25 & 0.12 & 6.42 & 0.35 \\
\hline
\end{tabular}

from the standardization of the absolute error

$$
e_{r, \mathrm{GABA}}^{(i)}=\left|y_{\mathrm{GABA}}^{(i)}-\hat{y}_{\mathrm{GABA}}^{(i)}\right| / y_{\mathrm{GABA}}^{(i)}, \quad e_{r, \mathrm{DA}}^{(i)}=\left|y_{\mathrm{DA}}^{(i)}-\hat{y}_{\mathrm{GABA}}^{(i)}\right| / y_{\mathrm{DA}}^{(i)},
$$

and the values for the six equations for both neurotransmitter systems are shown in Table 4 . The maximum of all relative errors is 0.32 for GABA and 0.35 for DA.

Table 4. The absolute and relative errors between measurement and model output for the three-neuron model with HFS primarily effecting GABAergic neurons.

\begin{tabular}{lccccc}
\hline & \multicolumn{2}{c}{ GABA } & & \multicolumn{2}{c}{ DA } \\
\cline { 2 - 3 } \cline { 6 - 7 } & Absolute $(\mathrm{nM})$ & Relative & & Absolute (nM) & Relative \\
\hline CTRL & 2.05 & 0.32 & & 0.00 & 0.00 \\
VER & 0.00 & 0.00 & & 3.41 & 0.13 \\
VER + HFS & 2.83 & 0.14 & & 1.63 & 0.09 \\
VER + HFS + BIC & 0.57 & 0.02 & & 0.10 & 0.00 \\
VER + HFS + SLP & 0.00 & 0.00 & & 3.06 & 0.17 \\
VER + HFS + SLP + SCH & 2.25 & 0.12 & & 1.38 & 0.07 \\
\hline
\end{tabular}


The identified coefficients provide acceptable errors only for the GABAergic system. Inhibitory and excitatory nature of interactions for the GABAergic system corresponds to the hypotheses in Section 2.1. This shows that GABA neurons within the striatum exclusively interact with DA neurons. Analysing the sign of coefficients, (self-)inhibition induced by increased GABA outflow under HFS validates the assumptions for inhibitory and excitatory effects. The two-fold effects of the dopaminergic system on GABA neurons (inhibitory and excitatory) are also well supported by coefficients found for the GABAergic neuron family.

The results for the dopaminergic system, however, show large errors if identification is based on the model in Figure 2. Furthermore, the sign of coefficients does not accord to the assumptions of self-inhibition $\left(N_{4}\right)$ and the inhibitory effect of GABAergic neurons $\left(N_{3}\right)$ on the dopaminergic system.

This might be due to different reasons:

(i) Insufficient modelling within the two-neuron model, e.g. lack of certain interactions.

(ii) Insufficient modelling of the measured results by a simple two-neuron model, e.g. more neuron groups have to be included.

\section{Three-neuron model}

\subsection{Modelling}

Based on the insufficient results given above, different additional pathways (e.g. self-excitation of the GABAergic and dopaminergic system) have been tested. Addition of interactions does not lead to significantly better results. Therefore, the two-neuron model is extended to a threeneuron model. Glutamatergic corticostriatal and thalamostriatal pathways are assumed to have an excitatory influence on the dopaminergic system within the striatum [13]. GLU modulates DA outflow by presynaptic excitation of neurons from the substantia nigra via $N$-methyl-Daspartic acid (NMDA) receptors [15] (Figure 3). Consideration of GLU into our theoretical model can consequently be achieved as another input to the dopaminergic system. This input is assumed to be positive (excitatory) and constant as it is not affected by HFS and the respective antagonists. The augmented three-neuron model is shown in Figure 4.

\subsection{Mathematical identification}

\subsubsection{HFS primarily affects GABAergic neurons}

The respective measurement scenarios resulting in different interactions between GABA and DA set up a set of dissimilar equations for the GABAergic system (Equation (3)) and the dopaminergic system (Equation (10)) as in Section 2.2. This modelling approach holds under

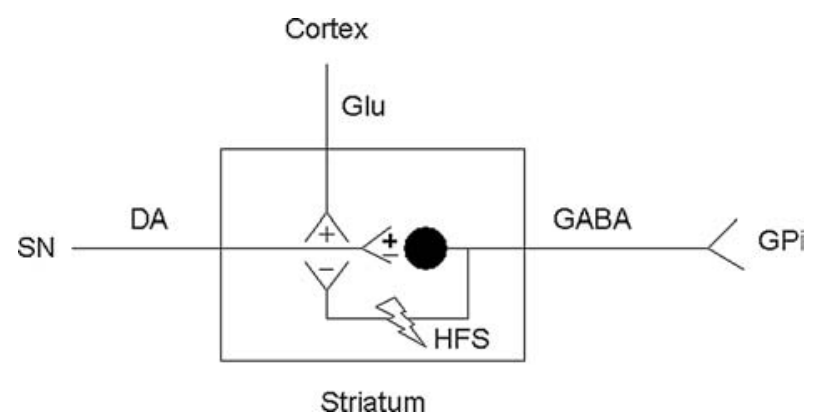

Figure 3. Consideration of GLU, excitatory to dopaminergic neurons. GPi: internal globus pallidus, SN: substantia nigra. 


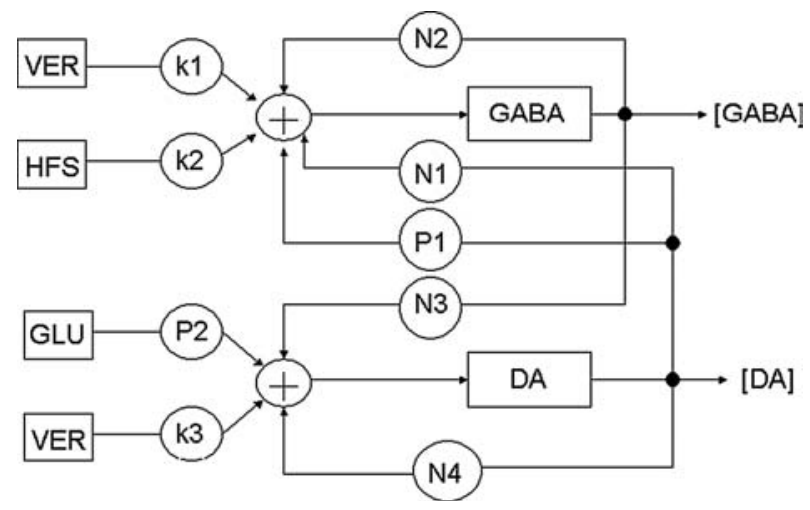

Figure 4. Three-neuron model of the GABA-DA relation under HFS. HFS primarily affects the GABAergic system. The coefficients of modulation are circled and network components are indicated by rectangles. Inhibition is given by factors $N$, excitation by $P$.

the assumption that HFS affects the dopaminergic system indirectly via presynaptic $\mathrm{GABA}_{A}$ receptors. None of the considered conditions inhibits the connection between DA and GLU in the model labelled with the factor $P_{2}$,

$$
\begin{aligned}
N_{3} \mathrm{GABA}^{(1)}+N_{4} \mathrm{DA}^{(1)}+P_{2} \mathrm{GLU}^{(1)} & =\mathrm{DA}^{(1)}, \\
k_{3}+N_{3} \mathrm{GABA}^{(2)}+N_{4} \mathrm{DA}^{(2)}+P_{2} \mathrm{GLU}^{(2)} & =\mathrm{DA}^{(2)}, \\
k_{3}+N_{3} \mathrm{GABA}^{(3)}+N_{4} \mathrm{DA}^{(3)}+P_{2} \mathrm{GLU}^{(3)} & =\mathrm{DA}^{(3)}, \\
k_{3}+N_{4} \mathrm{DA}^{(4)}+P_{2} \mathrm{GLU}^{(4)} & =\mathrm{DA}^{(4)}, \\
k_{3}+N_{3} \mathrm{GABA}^{(5)}+N_{4} \mathrm{DA}^{(5)}+P_{2} \mathrm{GLU}^{(5)} & =\mathrm{DA}^{(5)}, \\
k_{3}+N_{3} \mathrm{GABA}^{(6)}+P_{2} \mathrm{GLU}^{(6)} & =\mathrm{DA}^{(6)} .
\end{aligned}
$$

The absolute and relative errors of this three-neuron model are determined in the same way as in the two-neuron model and are shown in Table 4 . The maximum of the absolute error is 2.83 in the case of GABA and 3.41 for DA. The maximum of all relative errors is for GABA 0.32 and

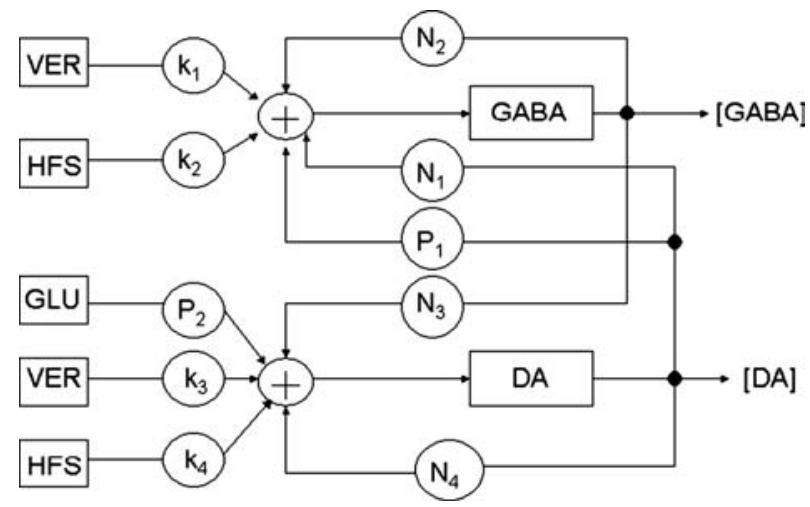

Figure 5. Three-neuron model of the GABA-DA relation under HFS. The coefficients of modulation are circled and network components are indicated by rectangles. Inhibition is given by factors $N$, excitation by $P$. 
for DA 0.17. Table 2 shows the identified interaction coefficients if mean concentrations of GABA and DA from Table 1 are used. As results for the modelling according to Figure 4 still do not confirm the proposed inhibitory and excitatory interactions between GABA, DA and GLU in the rat striatum, an augmented variant of the three-neuron model is tested.

\subsubsection{HFS affects GABAergic and dopaminergic neurons}

The neurotransmitter dependence model presented in Section 3.2.1 is extended by the assumption that HFS affects dopaminergic neurons directly (Figure 5). The resulting equations for the dopaminergic system are given by

$$
\begin{aligned}
N_{3} \mathrm{GABA}^{(1)}+N_{4} \mathrm{DA}^{(1)}+P_{2} \mathrm{GLU}^{(1)} & =\mathrm{DA}^{(1)}, \\
k_{3}+N_{3} \mathrm{GABA}^{(2)}+N_{4} \mathrm{DA}^{(2)}+P_{2} \mathrm{GLU}^{(2)} & =\mathrm{DA}^{(2)}, \\
k_{3}+k_{4}+N_{3} \mathrm{GABA}^{(3)}+N_{4} \mathrm{DA}^{(3)}+P_{2} \mathrm{GLU}^{(3)} & =\mathrm{DA}^{(3)}, \\
k_{3}+k_{4}+N_{4} \mathrm{DA}^{(4)}+P_{2} \mathrm{GLU}^{(4)} & =\mathrm{DA}^{(4)}, \\
k_{3}+k_{4}+N_{3} \mathrm{GABA}^{(5)}+N_{4} \mathrm{DA}^{(5)}+P_{2} \mathrm{GLU}^{(5)} & =\mathrm{DA}^{(5)}, \\
k_{3}+k_{4}+N_{3} \mathrm{GABA}^{(6)}+P_{2} \mathrm{GLU}^{(6)} & =\mathrm{DA}^{(6)}
\end{aligned}
$$

which leads to the results of the least-square solution shown in Table 2.

Table 2 shows the results for the coefficients if mean concentrations of GABA and DA from Table 1 are used. The absolute and relative errors of this three-neuron model are shown in Table 5. The maximum value of the absolute error is 2.83 for GABA and 1.55 for DA. The maximum value of all relative errors is 0.32 for GABA and 0.09 for DA.

Identification by means of the mathematical description of a three-neuron model shown in Figure 5 confirms the proposed inhibitory and excitatory interactions between GABA, DA and GLU in the rat striatum under HFS. Most importantly, the GABAergic system shows the strong interactions with the dopaminergic system but does not interact with the glutamatergic system. The dopaminergic system in the striatum, however, is stimulated by glutamatergic neurons. HFS is shown to excite the GABA neurons while having inhibitory effects on the DA neurons. The resulting outflow of the respective neurotransmitters leads to a steady state. VER increases the neurotransmitter concentration as assumed in the model. GABA is amplified with a factor of 9.12 and DA with 11.48. HFS amplifies the effect of VER on GABA while the DA outflow decreases under HFS $\left(k_{4}=-6.48\right)$.

Table 5. The absolute and relative errors between measurement and model output for the three-neuron model with HFS affecting GABAergic and dopaminergic neurons.

\begin{tabular}{lccccc}
\hline & \multicolumn{2}{c}{ GABA } & & \multicolumn{2}{c}{ DA } \\
\cline { 2 - 3 } \cline { 6 - 6 } & Absolute $(\mathrm{nM})$ & Relative & & Absolute (nM) & Relative \\
\hline CTRL & 2.05 & 0.32 & & 0.00 & 0.00 \\
VER & 0.00 & 0.00 & & 0.00 & 0.00 \\
VER + HFS & 2.83 & 0.14 & & 0.91 & 0.05 \\
VER + HFS + BIC & 0.57 & 0.02 & & 0.45 & 0.02 \\
VER + HFS + SLP & 0.00 & 0.00 & & 1.55 & 0.09 \\
VER + HFS + SLP + SCH & 2.25 & 0.12 & & 0.19 & 0.01 \\
\hline
\end{tabular}




\subsubsection{Sensitivity analysis}

The identified interaction coefficients $x$ which are obtained using the proposed model describe in which way GABAergic and dopaminergic neurons are qualitatively linked. Results from identification confirm the proposed connections in the three-neuron model (Figure 5). Moreover, the solution $x$ shows the quantitative effect of each individual connection $k_{1}, k_{2}, k_{3}, k_{4}, N_{1}, N_{2}, N_{3}$, $N_{4}, P_{1}$ and $P_{2}$ with respect to the average concentrations $y$. Up to now, however, the influence of small disturbances of the measured concentrations $y$ on the identification results $x$ have not been examined. Simulations show that if the mean values of $y$ are varied by $8.53 \%(1 / 8 \sigma)$, the result $x$ changes by maximally $12.58 \%$. Here, $\sigma$ denotes the standard deviation of the outflow values given in Table 1. If one permits larger deviations of the mean values $y$, larger changes of the identified coefficients $x$ are obtained (Table 6). These results show a high sensitivity of the identified coefficients versus changes of the measured outflow concentrations. Consistency with the proposed neurological model, however, is given as even in the case of a deviation of $68.27 \%$, the proposed inhibitory and excitatory interactions are verified by the signs of the identified coefficients.

\section{Discussion}

Deep brain stimulation applied at HFS in diverse regions of neuronal networks alleviates symptoms of neurodegenerative and neuropsychiatric disorders [10,11]. Although the complex mechanism of action of HFS is still not known, it has been extended to more and more indications [6,22]. Thus, it is necessary to look for a theoretical model to predict the effect of HFS in functional units of neurotransmission. In former neurochemical experiments, slices of the rat striatum were stimulated by the electrical pulses with $130 \mathrm{~Hz}$ and incubated in superfusion chambers [17]. This in vitro model was used to study the effect of HFS on an integrated neural network with functioning glia and neurons without connections to other brain regions [18]. The previous experimental results led to the assumption of a HFS-evoked modulation of a GABA-DA circuit pre-activated by the sodium channel opener VER. Since there was no alteration of GLU, it was pointed out that striatal slices would be suitable to study a two-component model consisting of GABAergic neurons and dopaminergic nerve terminals [16]. On the basis of these neurochemical results, we developed a theoretical model that allows quantitative determination of neural interactions in the rat striatum under HFS. Six hypotheses about GABA and DA modulation were derived from the past in vitro experiments to calculate a simple two-neuron model that could explain the effect of HFS in the rat striatum. The analytical two-neuron model, however, did not validate all assumptions made for the neural network. Thus, complementary to the neurochemical experiments, the analytical model led mainly to the following new insights.

Establishing a three-neuron model by enclosing a constant excitatory glutamatergic system is necessary to achieve a mathematical simulation that yielded good correspondence with the measurements for both, the GABAergic and dopaminergic system. The results are obtained

Table 6. Sensitity analysis for the identified interactions coefficients if measured GABA, DA, and GLU outflow is varied, $\sigma$ : standard deviation of measured outflow (nM).

\begin{tabular}{lcc}
\hline Outflow change with respect to mean value $(\%)$ & Outflow change $(\sigma)$ & Coefficient change $(\%)$ \\
\hline 8.53 & $1 / 8$ & 12.58 \\
17.07 & $1 / 4$ & 25.87 \\
34.14 & $1 / 2$ & 54.8 \\
68.27 & 1 & 123.89 \\
\hline
\end{tabular}


assuming a static behaviour of the neural network with linear interactions. Identification of five unknown interaction coefficients from six measurement scenarios leads to a weakly overdetermined system. This establishes a high sensitivity of the quantitative results to the quantitative values of the measurement thus reducing the reliability of the identification. The number of measurement scenarios, however, is limited by the biochemical options of modifying the neural network (e.g. by introduction of certain antagonists as bicuculline). Thus, the proposed system identification is limited to this weak over-determined nature and results have to be interpreted under these premises. Sensitivity analysis shows that the quantitative values of the interaction coefficients strongly depend on variations of the identification input values (e.g. outflow values). Results, however, confirm the qualitative nature of the neurological model as coefficients obtained under large deviations of the measured outflow concentrations still correspond to the proposed nature of interactions. According to the conducted neurochemical experiments, the identified coefficients could show that HFS features a stimulating effect on GABAergic neurons while having additionally an inhibitory effect on DA neurons. Interestingly, the analytical results led to the conclusion that inhibition of the dopaminergic system is not only caused indirectly by the increased activity of inhibitory GABAergic neurons but also directly by HFS. This is in contrast to our previous findings that DA outflow after HFS by $130 \mathrm{~Hz}$ is completely antagonized by $\mathrm{GABA}_{A}$ receptor blockade in slices of the rat striatum [17].

In conclusion, the findings of our former neurochemical experiments completed by the actual analytical evidences draws a more complex picture of neural action in the striatum under HFS.

In our analytical model, we found low errors if glutamatergic neurons have a direct excitatory influence on dopaminergic but not on GABAergic neurons. This result seems to confirm the assumption of Johnson et al. [13] who supposed a direct glutamatergic influence on dopaminergic terminals via NMDA receptors. Otherwise, another discussion about a more complex structure of a synapse with involvement of all three neurotransmitters within one spine can be found in further publications. The hypothesis describes both, glutamatergic and dopaminergic afferents synapse on the same spiny GABAergic neurons in the striatum [20]. Thereby, they possibly arrange asymmetric synapses with an excitatory glutamatergic input on the spine head and a dopaminergic synapse at its base [7,9]. Studies of individual spines led to the opinion that dopaminergic neurons are involved in the regulation of the signal from the corresponding spine head to its dendrite [2]. This contradiction between the calculated results and different neuroanatomic hypotheses could be resolved if intercellular communication of neurons is not only based on 'wiring' but also on 'volume transmission' [1,2]. This means that DA is released from the synaptic cleft as a centre of a sphere of influence followed by free diffusion into the surrounding extracellular tissue to its receptor targets. So, on the one hand, there is a 'wiring transmission' of GLU on spiny GABAergic neurons; on the other hand, there is a 'volume transmission' that led to a diffuse DA signal. Arbuthnott and Wickens [2] support the hypothesis of a non-selective signal mode based on 'volume transmission' of dopaminergic neurons in the striatum. They pointed out that this 'volume transmission' mode could achieve selectivity by temporal conjunction of diffuse DA signal and glutamatergic neural activity. We conclude that this timing of DA release in relation to glutamatergic activity is expressed by the described excitatory effect of GLU on dopaminergic neurons in our analytical model. So, the used model is able to define functional units of neurotransmission, but of course they cannot resolve the anatomical linkage between dopaminergic, glutamatergic and GABAergic neurons. Furthermore, the principle of timed 'volume transmission' could explain the contradictoriness of the necessity of GLU in the analytical model although the glutamatergic transmission is defined as constant according to our previous neurochemical experiments [16]. But it is not known how the timing of the GLU and DA transmission is initiated. Probably, activation of NMDA receptors localized on nigrostriatal dopaminergic nerve terminals which can modulate 
GABA release by directly activating its receptors on GABAergic neurons or indirectly through its receptors on corticostriatal glutamatergic terminals plays an important role $[12,19]$. In conclusion, it seems to be encouraging to develop a theoretical model based on neurochemical experiments and further mathematical processing to characterize functional units within neuronal networks. The aim of such a model is to obtain a calculated prediction about the effect of HFS and consecutively to find the best side of electrode implantation. So, the next step would be to look at an interference of HFS with a pathological altered neuronal network. Further neurochemical and analytical experiments are planned to comprehend a complex neuronal network that pictures not only physiological but also pathophysiological functional conditions under HFS. Finally, the characterization of different functional circuits under HFS should help in tackling the difficult issue, which patients with defined neurodegenerative or neuropsychiatric disorders would profit from an electrical stimulation of which points within cortico-basal ganglia-thalamo-cortical loops at which time of pathogenesis.

\section{Conclusion}

This contribution presented the modelling and numerical identification of the HFS evoked neuronal interactions of the neurotransmitters GABA, DA, and GLU in the rat striatum. Based on the neurochemical analysis, outflow values of the respective neurotransmitters under different measurement scenarios including the effect of receptor antagonists bicuculline, sulpiride, and SCH-23390 under HFS were determined. Interpreting the quantitative measurement values, a linear two-neuron model describing the mutual interaction between GABA and DA was derived. Interaction coefficients within this model were identified by a least-squares approach providing qualititative (e.g. inhibition or excitation) and quantitative information. Errors between model output and measured values were used to demonstrate the quality of the model. As error values did not provide acceptable errors and the sign of the identified coefficients did not correspond to previously made assumptions, the initial two-neuron model was firstly extended to a threeneuron model including GLU and secondly refined to reflect HFS affection of dopaminergic neurons. For the refined three-neuron model, numerical identification results show acceptable absolute and relative errors and validate assumptions made on the interaction of the different neurons. Results were subsequently interpreted reflecting different neuroanatomical hypotheses. To conclude, the modelling process led to an analytical model of the interactions within distinct neural network components of the rat striatum providing new insights into functional brain modelling. Further experiments are planned to gain insights into the possibility to develop a theoretical model that predicts the mechanism of HFS within functional units of neurotransmission for different neurodegenerative and neuropsychiatric disorders. This should lead to a refinement of the management of patients in respect of the best selection of patients that would profit from functional neurosurgery and to a better comprehension of the neurosurgical technique.

\section{Note}

1. L. Ramrath and J. Levering contributed equally to this work.

\section{References}

[1] L. Agnati, M. Zoll, I. Strömberg, and K. Fuxe, Intercellular communication in the brain: wiring versus volume transmission, Neuroscience 69 (1995), pp. 711-726.

[2] G. Arbuthnott and J. Wickens, Space, time and dopamine, Trend Neurosci. 30 (2007), pp. 62-69. 
[3] A. Benabid, What the future holds for deep brain stimulation, Exp. Rev. Med. Dev. 4 (2007), pp. 895-903.

[4] A. Benabid, A. Benazzouz, and P. Pollak, Mechanisms of deep brain stimulation, Mov. Disord. 17 (2002), pp. 73-74.

[5] A. Benazzouz and M. Hallet, Mechanisms of deep brain stimulation, Neurology 55 (2000), pp. 13-16.

[6] J. Dowling, Deep brain stimulation: current and emerging indications, Mo. Med. 105 (2008), pp. 424-428.

[7] T. Freund, J. Powell, and A. Smith, Tyrosine hydroxylase-immunoreactive boutons in synaptic contact with identified striatonigral neurons, with particular reference to dendritic spines, Neuroscience 13 (1984), pp. 1189-1215.

[8] R. Gross and A. Lozano, Advances in neurostimulation for movement disorders, Neurol. Res. 22 (2000), pp. 247-258.

[9] P. Groves, J. Linder, and S. Young, 5-Hydroxydopamine-labeled dopaminergic axons: threedimensional reconstructions of axons, synapses and postsynaptic targets in rat neostriatum, Neuroscience 58 (1994), pp. 593-604.

[10] D. Guehl, A. Benazzouz, B. Aouizerate, E. Cuny, J. Rotgé, A. Rougier, J. Tignol, B. Bioulac, and P. Burbaud, Neuronal correlates of obsessions in the caudate nucleus, Biol. Psychiatry 63 (2008), pp. 557-562.

[11] C. Hammond, R. Ammari, B. Bioulac, and L. Garcia, Latest view on the mechanism of action of deep brain stimulation, Mov. Disord. (2008), Epub ahead of print.

[12] T. Hanania and K. Johnson, Regulation of NMDA-stimulated [ $\left.{ }^{1} 4 C\right] G A B A$ and $\left[{ }^{3} H\right]$ acetylcholine release by striatal glutamate and dopamine receptors, Brain Res. 844 (1999), pp. 106-117.

[13] K. Johnson and Y.J. Jeng, Pharmacological evidence dor N-methyl-D-aspartate receptors on nigrostriatal dopaminergic nerve terminals, Can. J. Physiol. Pharmacol. 69 (1991), pp. 1416-1421.

[14] P. Krack, A. Benazzouz, P. Pollak, P. Limousin, B. Piallat, D. Hoffmann, J. Xie, and A. Benabid, Treatment of tremor in Parkinson's disease by subthalamic nucleus stimulation, Mov. Disord. 13 (1998), pp. 907-914.

[15] M. Krebs, J. Desce, M. Kemel, C. Gauchy, G. Godeheu, A. Cheramy, and J. Glowinski, Glutamatergic control of dopamine release in the rat striatum: evidence for presynaptic N-methyl-Daspartate receptors on dopaminergic nerve terminals, J. Neurochem. 56 (1991), pp. 81-85.

[16] T. Li, A. Thuemen, and A. Moser, Modulation of a neuronal network by electrical high frequency stimulation in striatal slices of the rat in vitro, Neurochem. Int. 48 (2006), pp. 83-86.

[17] A. Moser, A. Gieselberg, B. Ro, C. Keller, and F. Qadri, Deep brain stimulation: response to neuronal high frequency stimulation is mediated through $G A B A(A)$ receptor activation in rats, Neurosci. Lett. 341 (2003), pp. 57-60.

[18] K. Reid, H. Edmonds, A. Schurr, M. Tseng, and C. West, Pitfalls in the use of brain slices, Prog. Neurobiol. 31 (1988), pp. 1-18.

[19] R. Schwarcz, I. Creese, J. Coyle, and J. Snyder, Dopamine receptors localized on cerebralcortical afferents to rat corpus striatum, Nature 271 (1987), pp. 766-768.

[20] A. Smith and J. Bolam, The neural network of the basal ganglia as revealed by the study of synaptic connections of identified neurons, Trends Neurosci. 13 (1990), pp. 259-265.

[21] V. Tronnier, W. Fogel, M. Krause, M. Bonsanto, J. Tronnier, A. Heck, K. Munkel, and S. Kunze, High frequency stimulation of the basal ganglia for the treatment of movement disorders: current status and clinical results, Minim. Invasive Neurosurg. 45 (2002), pp. 91-96.

[22] J. Vitek, Deep brain stimulation: how does it work?, Cleve. Clin. J. Med. 75 (2008), pp. 59-65. 


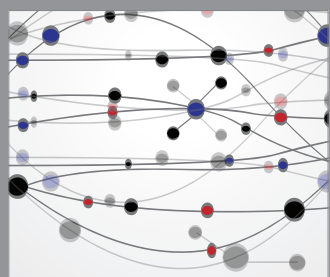

The Scientific World Journal
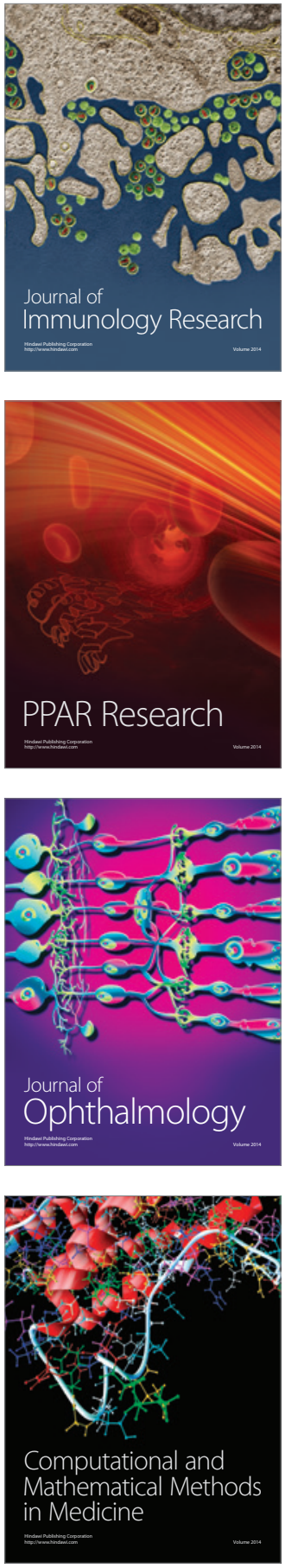

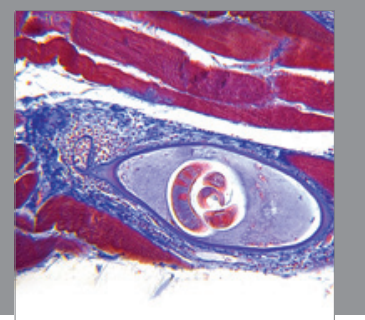

Gastroenterology

Research and Practice
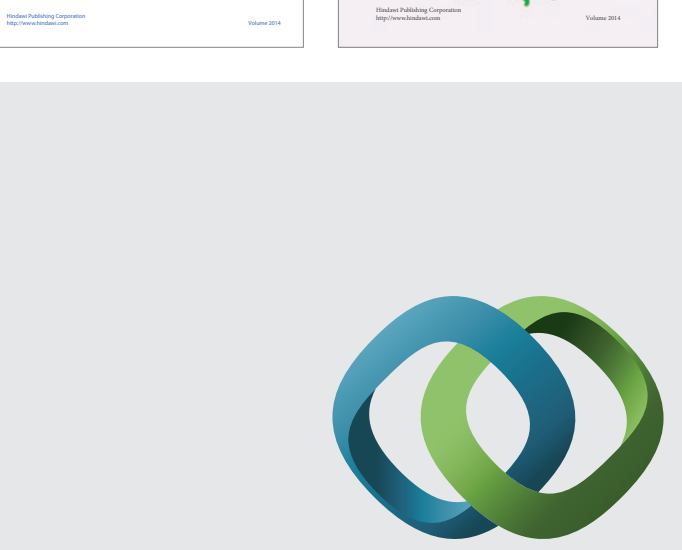

\section{Hindawi}

Submit your manuscripts at

http://www.hindawi.com
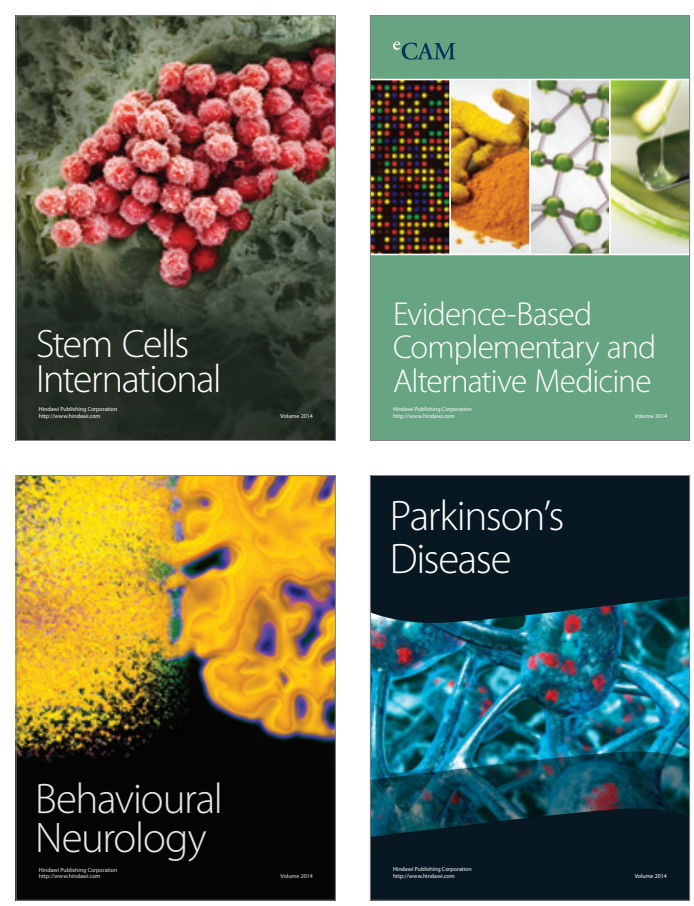

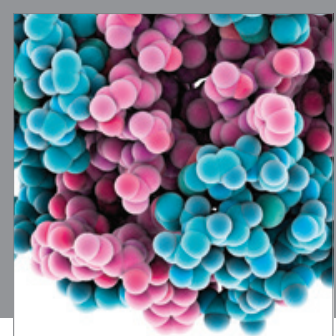

Journal of
Diabetes Research

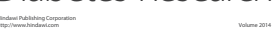

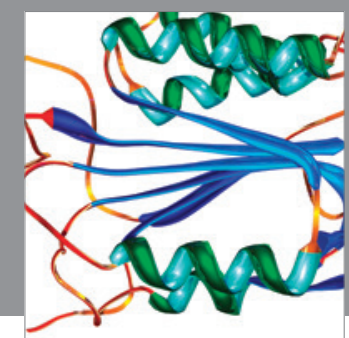

Disease Markers
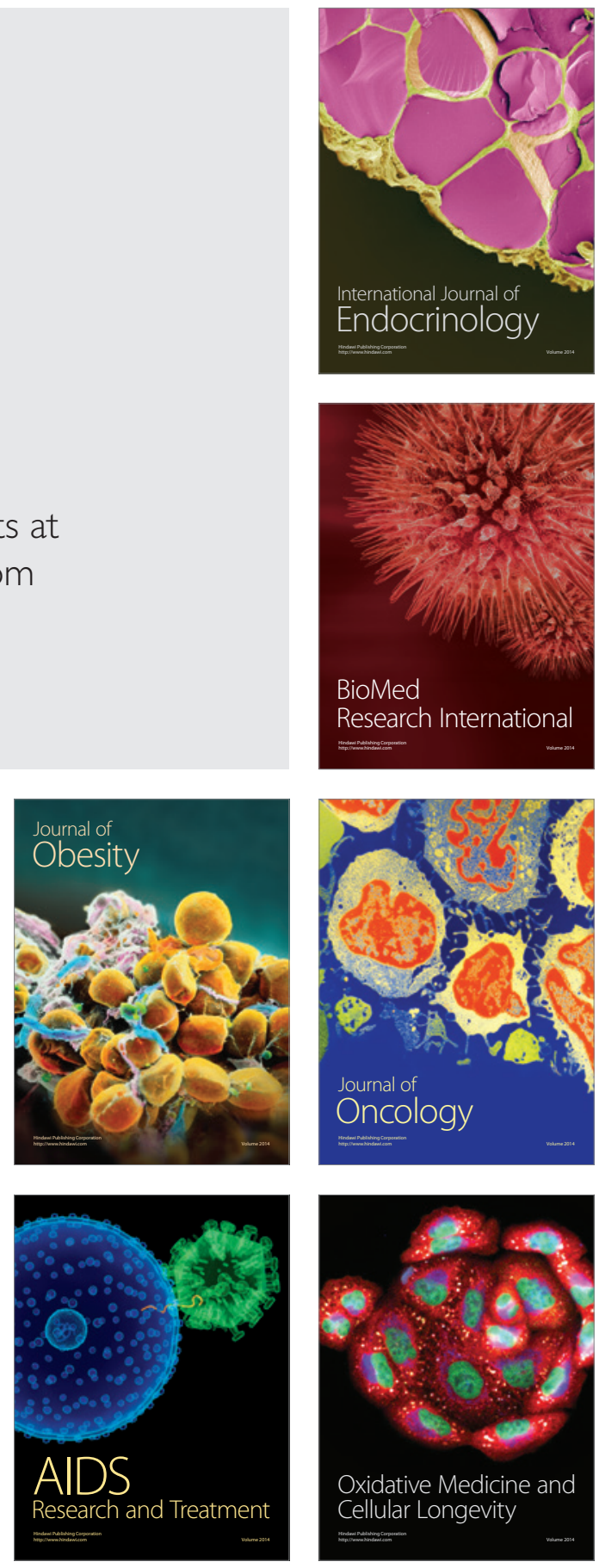